\title{
Septal Neurons in Barrel Cortex Derive Their Receptive Field Input from the Lemniscal Pathway
}

\author{
Takahiro Furuta, ${ }^{1}$ Takeshi Kaneko, ${ }^{1,2}$ and Martin Deschênes ${ }^{3}$ \\ ${ }^{1}$ Department of Morphological Brain Science, Graduate School of Medicine, Kyoto University, Kyoto 606-8501, Japan, ${ }^{2}$ Core Research for Evolution Science \\ and Technology, Japan Science and Technology Agency, Kawaguchi 332-0012, Japan, and ${ }^{3}$ Centre de Recherche, Université Laval Robert-Giffard, Québec \\ City, Canada G1J 2G3
}

Barrel-related circuits in the somatosensory cortex of rodents process vibrissal information conveyed through the lemniscal pathway. Yet, the origin of vibrissal input to interbarrrel regions (septa) remains an unsettled issue. A recurring proposal that never received conclusive experimental support is that septa-related circuits process paralemniscal inputs conveyed through the posterior group of the thalamus. Here we show that the receptive field of septal cells is independent of paralemniscal inputs, and that septal cells derive their receptive field input from neurons in the dorsal part of the thalamic barreloids. This result provides the missing piece of evidence for a separate pathway of vibrissal information that projects to septal columns of the barrel cortex.

\section{Introduction}

The primary somatosensory cortical area (S1) in rodents comprises two intercalated cytoarchitectonic divisions: a granular zone characterized by dense cellular aggregates in layer 4 (i.e., barrels), and a dysgranular zone that consists of interbarrel septa and regions in the immediate surrounding of the barrel field. The collections of cells vertically aligned with barrels and septa are commonly referred to as barrel and septal columns, respectively. A number of studies indicate that barrel and septal columns represent two separate streams of vibrissa information processing that differ in their response patterns and anatomical connections (Kim and Ebner, 1999; for review, see Alloway, 2008). Latency analyses of vibrissal responses further revealed that septal neurons respond to whisker deflection at virtually the same time as barrel neurons, but several milliseconds before supragranular neurons (Brumberg et al., 1999), which indicates that both populations of cells must derive their receptive field input from the thalamus. Whereas there is consensus that barrel-related circuits process lemniscal inputs that transit through thalamic barreloids of the ventral posterior medial nucleus (VPM), septa-related circuits are often considered to process paralemniscal inputs conveyed through the posterior nuclear complex of the thalamus (Po). However, recent studies have shown that Po can hardly be responsible for vibrissal responses of septal cells in anesthetized animals, because sensory transmission through this nucleus is normally impeded by feedforward inhibition that arises from the zona incerta (Trageser and Keller, 2004; Lavallée et al., 2005).

\footnotetext{
Received Nov. 8, 2008; revised Feb. 16, 2009; accepted Feb. 19, 2009.

This work was supported by grants from Takeda Science Foundation and from the Ministry of Education, Science, Sports, and Culture of Japan (Grant 20700316) to T.F., and by the Canadian Institute of Health Research Grant MT-5877 (M.D.).

Correspondence should be addressed to Martin Deschênes at the above address. E-mail: martin.deschenes@crulrg.ulaval.ca.

DOI:10.1523/JNEUROSCI.5393-08.2009

Copyright $\odot 2009$ Society for Neuroscience $\quad$ 0270-6474/09/294089-07\$15.00/0
}

This indicates that septal cells should derive their multiwhisker receptive field from the VPM itself.

Previous studies have identified three pathways of vibrissal information that transit through different sectors of the VPM: (1) a dorsal pathway that passes through the "head" of the barreloids, near Po, in which cells respond to multiple whiskers (Urbain and Deschênes, 2007); (2) a central pathway that transits through the "core" of the barreloids in which cells are primarily driven by a single whisker; and (3) a ventral pathway that passes through the ventral lateral part of the VPM (VPMvl) (i.e., the "tail" of the barreloids), in which cells respond to multiple whiskers (Pierret et al., 2000; Bokor et al., 2008). Whereas the dorsal and central pathways relay input from the principal trigeminal nucleus ( $P r V)$ to barrel cortex, the ventral pathway receives input from the interpolar trigeminal nucleus $(\mathrm{SpVi})$, and projects principally to the second somatosensory cortical area and less profusely to the dysgranular zone of S1 (Veinante and Deschênes, 1999; Pierret et al., 2000). In the present study, we sought to determine which of these pathways provides vibrissal input to septal neurons. Our results show that the receptive field of septal cells is independent of inputs from the SpVi and, therefore, from pathways that pass through Po and VPMvl. Furthermore, the anterograde labeling of single barreloid cells reveals that head cells project principally to septal regions, whereas core cells innervate mainly the homologous barrel. These results lead to the conclusion that the multiwhisker receptive field of septal cells derives primarily from their innervation by cells in the head of the barreloids.

\section{Materials and Methods}

Experiments were conducted in accordance with federally prescribed animal care and use guidelines. The Ethical Committee for Animal Use in Research (Laval University) and the Institute of Laboratory Animals, Graduate School of Medicine (Kyoto University) approved all experimental protocols.

Recording and labeling of septal neurons. Experiments on barrel cortex were performed in 19 male rats (250-300 g; Sprague Dawley) under 
ketamine (75 mg/kg, xylazine, $5 \mathrm{mg} / \mathrm{kg}$ ) anesthesia. The left facial nerve was cut, and the rat was placed in a stereotaxic apparatus. The animal breathed freely, and body temperature was maintained at $37.5^{\circ} \mathrm{C}$ with a heating pad controlled thermostatically. Throughout the experiment, a deep level of anesthesia was maintained (stage III-3; Friedberg et al., 1999) by additional doses of anesthetics $(20 \mathrm{mg} / \mathrm{kg}$ ketamine plus 0.3 $\mathrm{mg} / \mathrm{kg}$ xylazine, i.m.) given as needed to abolish reflex to sharp pinch of the hindlimbs.

Single units were recorded extracellularly in the right barrel cortex with micropipettes (tip diameter, $\sim 1 \mu \mathrm{m}$ ) filled with a solution of potassium acetate $(0.5 \mathrm{M})$ and biotinylated dextran amine (2\% BDA-10000; Invitrogen). The micropipette was lowered at an angle of $35^{\circ}$ from the vertical to record layer 4 cells (depth: $650-850 \mu \mathrm{m}$ below the pia) in barrels of the D and E rows (arcs 1-3) and in the intervening septa. By keeping the angle of penetration constant across the experiments for exploring the same cortical region, depth reading from the microdrive proved to be a reliable indicator of unit location in layer 4 . Because the aim of the experiments was to identify the ascending pathway that provides vibrissal input to septal neurons, we primarily sought to record multiwhisker cells, presuming that most were located in septa. When a multiwhisker unit was encountered in layer 4 , its receptive field size was assessed with a hand-held probe. Then a piezoelectric stimulator was used to confirm whether the cell responded to only one or to many whiskers. If a unit responded to more than one whisker to the piezoelectric stimulator (99\% confidence level with respect to spontaneous activity level estimated over a prestimulus time window of $100 \mathrm{~ms}$ ), it was considered as a multiwhisker cell. Stimuli consisted of ramp-and-hold deflections (rise/fall times, 2-10 ms; total duration, $50 \mathrm{~ms}$; amplitude, 400-800 $\mu \mathrm{m}$; interstimulus interval, $1.2 \mathrm{~s}$ ). Deflections were applied to the cut tip of the vibrissa ( $\sim 10 \mathrm{~mm}$ from the skin) in the direction that best drove the unit. Signals were amplified, bandpass filtered $(200 \mathrm{~Hz}$ to $3 \mathrm{kHz}$ ), and sampled at $10 \mathrm{kHz}$. Thereafter, the cell was labeled juxtacellularly according to the method described by Pinault (1996), or the recording site was labeled by an iontophoretic deposit of BDA ( 2 cases; 200 ms current pulses, half-duty cycle, $50 \mathrm{nA}$ for $5 \mathrm{~min}$ ). At the end of the recording session, animals were perfused under deep anesthesia with saline followed by a solution of $4 \%$ paraformaldehyde plus $0.2 \%$ glutaraldehyde in phosphate buffer $(0.1 \mathrm{M}, \mathrm{pH} 7.4)$.

Data analysis. Spike events elicited by vibrissa deflection were collected in peristimulus time histograms (PSTHs) of 20 responses with $1 \mathrm{~ms}$ bin width. Response magnitude was estimated as the mean number of spikes per stimulus within a time window of $20 \mathrm{~ms}$ after stimulus onset. We defined response onset as the first two consecutive bins (poststimulus) displaying counts that significantly exceeded ( $99 \%$ confidence interval) spontaneous activity levels estimated over a prestimulus time window of 100 ms. Data were analyzed with the Neuroexplorer (Plexon) and Excel (Microsoft) software. Results are reported as mean \pm SD.

Histology and cell reconstruction. After perfusion, brains were postfixed for $2 \mathrm{~h}$, cryoprotected in $30 \%$ sucrose, and cut at $60 \mu \mathrm{m}$ on a freezing microtome. Cortex was cut tangentially (13 brains) or coronally (4 brains). Sections were processed for cytochrome oxidase (CO) and BDA histochemistry according to standard protocols that were described in detail previously (Veinante and Deschênes, 1999).

Because tangential sections are not always parallel to the pia, the array of barrels was reconstructed from three to four serial sections using a low-magnification objective $(2.5 \times)$ to identify the rows and arcs of interest. A layer 4 neuron was classified as barrel or septa depending on whether or not the soma and most of its dendrites were in a CO-rich area.

Labeled neurons were drawn at $40 \times$ with a camera lucida; drawings were scanned, redrawn in Illustrator CS (Adobe Systems), and superimposed on the array of barrels after appropriate rescaling. Photomicrographs were taken with a Spot RT camera (Diagnostic Instruments) and imported in Photoshop 7.0 (Adobe Systems) for contrast and brightness adjustments.

Brainstem lesions. Before recording in barrel cortex, the spinal trigeminal tract was acutely lesioned in eight rats by passing direct current $(3$ $\mathrm{mA}, 3 \mathrm{~s}$ ) through a tungsten electrode (shaft diameter, $200 \mu \mathrm{m}$; tip diameter, $50 \mu \mathrm{m}$; deinsulated over $1 \mathrm{~mm}$ ). Lesion was made at the frontal plane $11.5 \mathrm{~mm}$ behind bregma (stereotaxic coordinates from the atlas of
Paxinos and Watson, 1998), and current was passed at multiple depths along two descents $400 \mu \mathrm{m}$ apart mediolaterally to fully destroy the targeted region.

Acute parasagittal brainstem lesions were made medial to the PrV in two rats by passing direct current $(2.0 \mathrm{~mA}, 2 \mathrm{~s})$ through a tungsten electrode as above. Lesions were made at several frontal planes (8.5-10 $\mathrm{mm}$ behind the bregma and $2.0 \mathrm{~mm}$ lateral to the midline; Paxinos and Watson, 1998), and at multiple depths (6.8 $-8.3 \mathrm{~mm}$ below the pia), to fully destroy the ascending axons of the PrV. In each lesioned rat the extent of the lesion was assessed on CO-stained frontal sections of the brainstem.

Recording and labeling of thalamic cells. Single thalamic units were recorded extracellularly with micropipettes $(0.5-1 \mu \mathrm{m})$ filled with a solution of potassium acetate $(0.5 \mathrm{~m})$ and $10 \% \mathrm{BDA}-3000$ (Invitrogen). Receptive field size and response properties were assessed with a handheld probe and with a piezoelectric stimulator using stimulus parameters similar to those used for testing cortical neurons. Then, cells were labeled juxtacellularly for 20-30 min (Pinault, 1996). After completing this protocol, the skin was sutured, rats were given analgesics (5 mg/kg Anafen), and they were returned to the animal facilities.

Two to three days later, rats were perfused under deep anesthesia with PBS followed by a fixative containing $4 \%$ paraformaldehyde and $0.5 \%$ glutaraldehyde in phosphate buffer $(0.1 \mathrm{M})$. After cryoprotection in $30 \%$ sucrose, barrel cortex was cut tangentially at $40 \mu \mathrm{m}$ on a freezing microtome. The thalamus was cut coronally at $70 \mu \mathrm{m}$. Sections were incubated overnight in ABC-Elite (Vector Laboratories) at room temperature. After three rinses in PBS, sections were reacted for 30 min with 2.5 $\mu \mathrm{m}$ biotinylated tyramine, $3 \mu \mathrm{g} / \mathrm{ml}$ glucose oxidase, and $2 \mathrm{mg} / \mathrm{ml}$ glucose. This was followed by incubation for $2 \mathrm{~h}$ in ABC-Elite, and peroxidase was revealed using nickel-diaminobenzidine as a substrate. Finally, sections were processed for CO.

The axonal arbor of barreloid cells was examined with a light microscope equipped with a computerized tracing system (Neurolucida, MicroBrightField). Labeled boutons were mapped with a CCD camera using a 30 -inch flat panel monitor connected to the Neurolucida system. This system had an intermediate magnification lens $(5 \times)$ in front of the CCD camera. Thus, with an objective lens of $40 \times$, the final magnification was $\sim 2000 \times$. The distribution of boutons in layer IV was mapped with respect to barrels and septa. Boutons were identified as en passant varicosities along axonal branches or as terminal beads at the tip of fine branches. Ultrastructural analysis has shown that bouton-like varicosities of VPM axons in barrel cortex make synaptic contacts onto cortical neurons (Lu and Lin, 1993).

The somatodendritic morphology of barreloid cells was reconstructed from serial sections with a camera lucida ( $40 \times$ objective). Branching structure of dendritic trees was assessed by Sholl analysis, and a $t$ test was used to estimate whether head and core cells display statistically significant difference in branch density.

\section{Results \\ Septal cells remain multiwhisker responsive after lesion of the SpVi}

Under the regimen of deep anesthesia used in the present experiments, layer 4 cells in barrel cortex were either clearly monowhisker or clearly multiwhisker responsive. As expected, monowhisker cells $(n=11)$ were regular-spiking neurons; they responded to whisker deflection with a mean number of $0.99 \pm$ 0.42 spike per deflection at a mean latency of $10.14 \pm 2.37 \mathrm{~ms}$. Five cells were labeled with BDA in normal and SpVi-lesioned rats, and all cell bodies were recovered in the barrel representing the corresponding whisker (Fig. S1, available at www.jneurosci. org as supplemental material).

Three layer 4 fast-spiking cells were also recorded in normal or SpVi-lesioned rats. They responded briskly to multiple whiskers (4-6 whiskers), but none was labeled. Given the small number of fast-spiking neurons, these data are not included in the present report. 

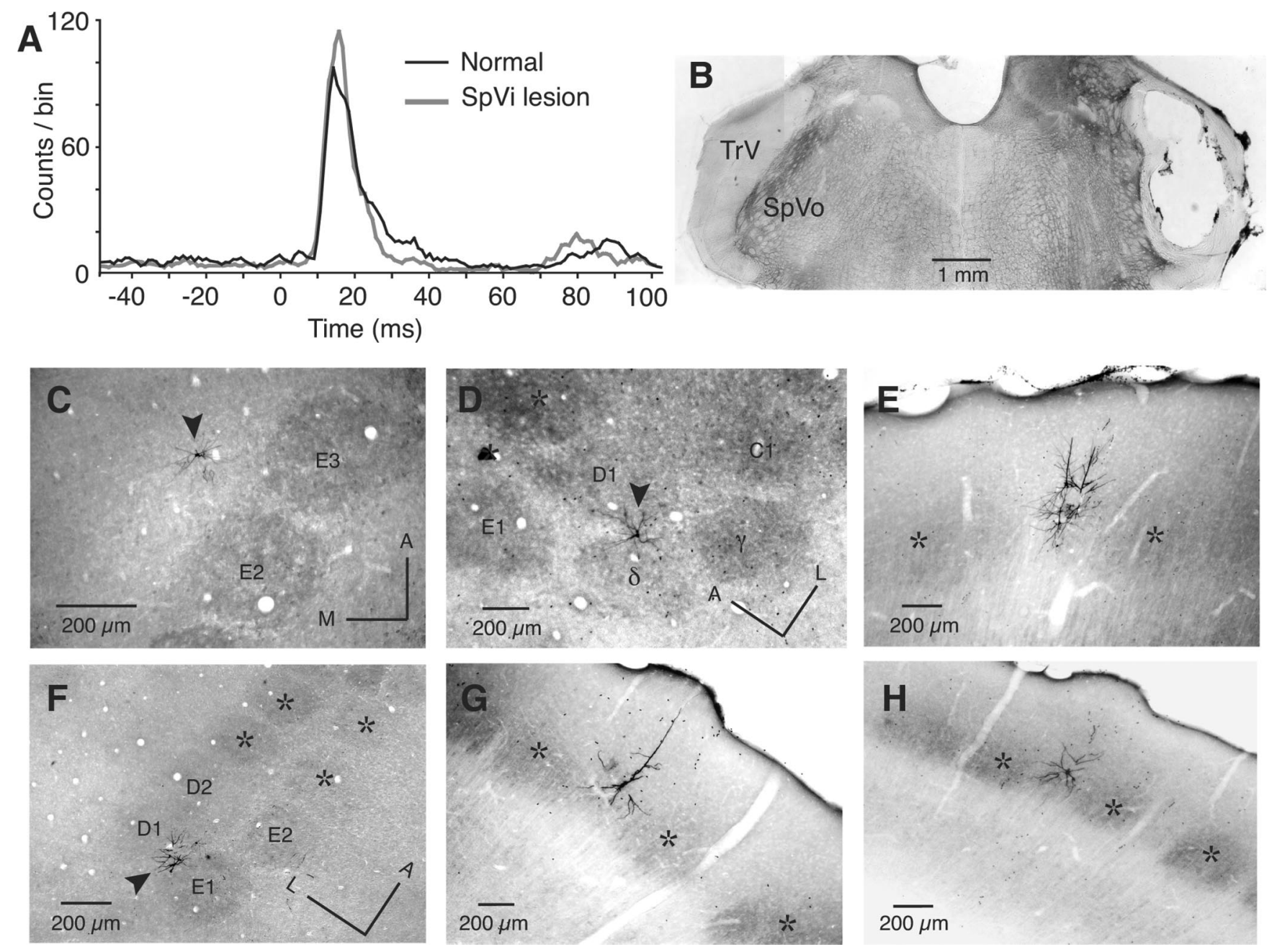

Figure 1. $\quad \boldsymbol{A}-\boldsymbol{H}$, Septal cells maintain their multiwhisker receptive field after lesion of sensory input to the paralemniscal pathway. $\boldsymbol{A}$, Population PSTHs of vibrissal responses recorded from septal neurons in normal rats ( $n=13$ cells; 42 vibrissae) and SpVi-lesioned rats ( $n=13$ cells; 43 vibrissae). Responses used to build PSTHs were obtained with $2 \mathrm{~ms}$ rise time deflections. $\boldsymbol{B}$, The extent of the trigeminal tract lesion is shown. $\mathbf{C}-\boldsymbol{H}$, Multiwhisker cells were located in septal columns (barrels are indicated by asterisks) as assessed by single-cell labeling $(\boldsymbol{C}, \boldsymbol{D}, \boldsymbol{G}, \boldsymbol{H})$, or local iontophoretic injection of BDA, which labeled a small cluster of cells $(\boldsymbol{E}, \boldsymbol{F})$. Cells labeled in normal rats $(\boldsymbol{C}-\boldsymbol{E})$ and SpVi-lesioned rats $(\boldsymbol{F}-\boldsymbol{H})$ are shown. Tangential $(\boldsymbol{C}, \boldsymbol{D}, \boldsymbol{F})$ and frontal $(\boldsymbol{E}, \boldsymbol{G}, \boldsymbol{H})$ sections of the barrel cortex were counterstained for $\mathrm{C} 0$. A, Anterior; L, lateral; $\mathrm{M}$, medial; $\mathrm{SpV}$, oralis division of the spinal trigeminal nucleus; $\mathrm{TrV}$, trigeminal tract.

The 20 regular spiking multiwhisker cells recorded in nine normal rats had, on average, a receptive field that included 3.54 vibrissae located in the first arcs of the D and E rows (Fig. S2, available at www.jneurosci.org as supplemental material). They responded to controlled whisker deflection with a mean number of $1.08 \pm 0.81$ spikes per deflection at a mean latency of $10.81 \pm$ $2.15 \mathrm{~ms}$ (Fig. 1A). Ten of these cells were labeled with BDA, and they were all recovered in septal columns (Fig. 1C-E; Fig. S1 A, available at www.jneurosci.org as supplemental material).

Lesion of the SpVi (8 rats) (Fig. $1 B$ ) did not change the likelihood of recording multiwhisker units in barrel cortex, nor did it produce any significant change in response properties. Just like in normal rats, regular-spiking multiwhisker cells $(n=18)$ responded, on average, to 4.35 vibrissae with a mean number of $1.25 \pm 0.70$ spikes per deflection (no significant difference with normal rats; $p=0.212 ; t$ test) (Fig. $1 A$ ). Nor did mean response latency significantly differed from that in normal rats (11.01 \pm $2,45 \mathrm{~ms} ; p=0.31 ; t$ test). Again, all multiwhisker units labeled in SpVi-lesioned rats $(n=7)$ were found within septal columns (Fig. $1 \mathrm{~F}-\mathrm{H}$; Fig. S1 $\mathrm{A}$, available at www.jneurosci.org as supplemental material).

Histological controls confirmed that lesions completely de- stroyed the descending limb of the trigeminal tract (Fig. 1B). Lesions also involved the caudal part of the oralis subnucleus, but they never extended rostrally beyond the emergence of the facial nerve. On the basis of these results, one has to conclude that the multiwhisker receptive field of septal cells relies on inputs from the PrV.

\section{PrV lesion abolishes vibrissal responses in the barrel cortex}

To further assess the potential contribution of the $\operatorname{PrV}$ to receptive field size in barrel cortex, we sampled 112 units through layers 3-5a (depth: $350-1000 \mu \mathrm{m}$ below the surface of the brain) after lesion of the lemniscal pathway in two rats. In sharp contrast with normal rats, in which virtually all neurons could be driven by manual deflection of the whiskers, the vast majority of these neurons (106 of 112 units, i.e., 95\%) were unresponsive to sensory stimuli (Fig. 2A). Parasagittal brainstem lesions were made medial to the $\operatorname{PrV}$ to sever crossing axons ascending toward VPM (Fig. $2 B-D$ ). As estimated from CO-stained sections of the brainstem, lesion extended rostrocaudally between frontal planes $8-10.5 \mathrm{~mm}$ behind bregma and throughout the depth of the brainstem. The lesion severed crossing axons that arise from the $\mathrm{PrV}$ and part of the PrV itself, but the 


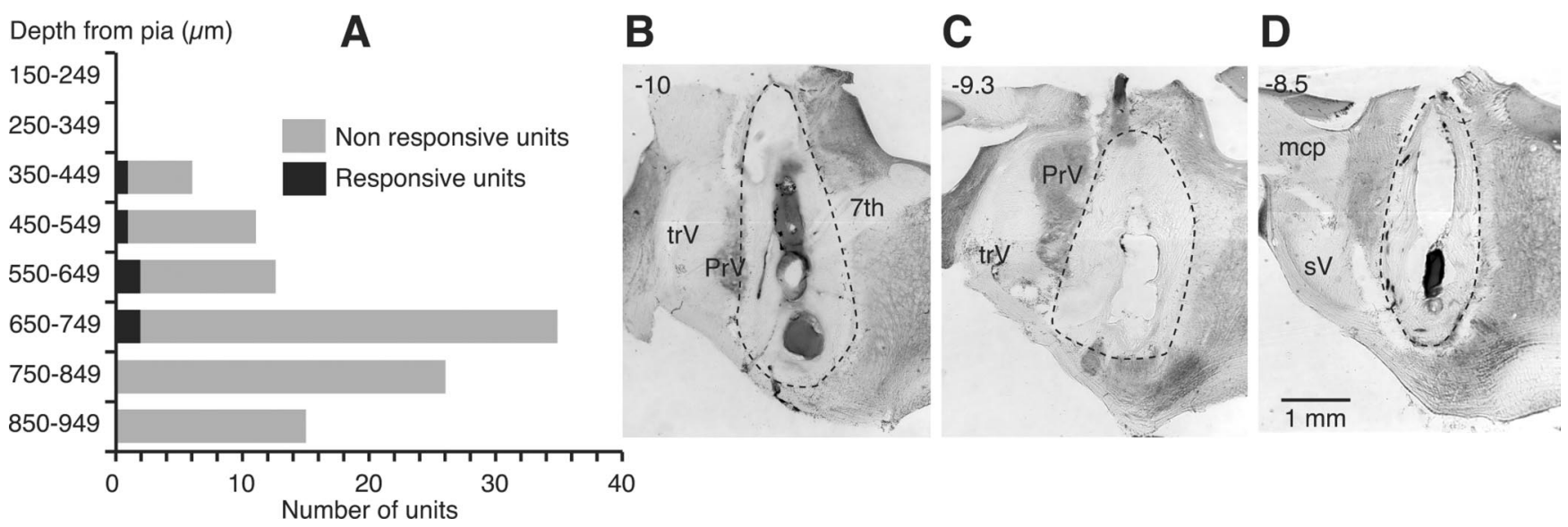

Figure 2. $A-D$, Vibrissal responses in barrel cortex are virtually abolished by lesion of the lemniscal pathway. Histogram in $\boldsymbol{A}$ shows the distribution of 112 units that were recorded in supragranular and granular layers of the barrel cortex in two PrV-lesioned rats. Only six of these units responded to whisker deflection. Photomicrographs in $\boldsymbol{B}-\boldsymbol{D}$ show the extent of the lesion that severed ascending axons from the PrV. Note that the lesion spared the trigeminal tract (TrV). Numbers in the upper left corner of the photomicrographs indicate the stereotaxic frontal planes behind bregma. 7th, Tractus of the facial nerve; mcp, middle cerebellar peduncle; sV, sensory root of the trigeminal nerve.

descending branches of primary afferents that travel in the trigeminal tract remained intact.

In sum, lesion experiments show that septal cells maintain their multiwhisker receptive field after lesion of the paralemniscal pathways (pathways that transit through Po and VPMvl), but together with barrel cells they become virtually unresponsive to whisker deflection after lesion of the lemniscal pathway. These results indicate that septal cells must derive their receptive field input from cells that reside in the core or head of the barreloids.

\section{Multiwhisker cells in the head of barreloids project to septa}

We recorded and labeled 19 barreloid cells that responded to controlled whisker deflection at a mean latency of $6.5 \pm 1.7$ ms. Seven neurons responded to multiple whiskers (average receptive field size, 3.43 vibrissae) (Fig. S2, available at www. jneurosci.org as supplemental material); they were located in the head of the barreloids, within a region $\sim 150 \mu$ m thick near Po (Fig. 3A-C) (additional examples are provided in Fig. S3, available at www.jneurosci.org as supplemental material). The other cells responded to a single vibrissa, and were generally located further away from the VPM/Po border, in the core of the barreloids.

Camera lucida reconstructions of labeled cells revealed that head and core cells display dendritic fields of approximately the same size (dendritic span, $\sim 250 \mu \mathrm{m}$ ), and that part of the dendritic field of head cells extends across the VPM/Po border (Fig. S4, available at www.jneurosci.org as supplemental material). Moreover Sholl analysis revealed a clear trend for head cells to generate more elaborate dendritic trees. Even with our small number of head cells, differences in the degree of branching nearly reached statistical significance ( $t$ test; $p=0.057$ ).

As they exit the thalamus, axons of head and core cells send collaterals in the reticular thalamic nucleus, traverse the striatum, and, on reaching barrel cortex, start dividing in the depth of layer 6. Daughter branches ascend through layer 6 , where they divide again, giving off small branches bearing boutons. Thicker branches further ascend and ramify profusely in layers 4 and 3, with a few branches reaching up to layer 2 .

Although axons of head and core cells display similar patterns of laminar innervation, the tangential distribution of terminal fields in layer 4 was strikingly different. Whereas boutons emitted by core cells were concentrated in a single barrel, those of head cells distributed preferentially in septa. Figure $3, D$ and $E$, shows a representative example of a head cell's axon whose branches divide abundantly in layer 4 , giving off thin branches and boutons in the septal region between rows B and C. Some branches also cut across barrels (barrel C2 in this particular case), where they give off boutons (Fig. 3F; see also Fig. S3G-J, available at www. jneurosci.org as supplemental material). In general, head cells generate more widespread terminal fields than do core cells, which extend preferentially in septa separating rows of vibrissa representation (Fig. $3 F-I$; see also Fig. S3, available at www. jneurosci.org as supplemental material). Although the axon of head and core cells gave off an approximately equal number of boutons in layer 4 (5166 \pm 684 versus $4852 \pm 861), 74.2 \pm 9.0 \%$ of boutons given off by head cells were found in septa $(n=5$ cells), whereas this proportion fell to $26.9 \pm 7.4 \%$ in the case of core cells ( $n=12$ cells). Both the size of boutons and their spacing along branches were approximately the same for both types of axons (size: $1.28 \pm 0.22 \mu \mathrm{m}, 658$ boutons; frequency: $0.20 \pm$ $0.02 / \mu \mathrm{m}$ for core cells; and $0.20 \pm 0.03 / \mu \mathrm{m}$ for head cells). Measures were made on five segments of axons per cell (mean segment length, $179 \mu \mathrm{m}$, for a total of 85 axon segments). These figures are remarkably similar to those reported by White et al. (2004), who found that thalamocortical afferents in mouse barrels make an average of 0.2 asymmetrical synapses/ $\mu \mathrm{m}$ length of axon.

\section{Discussion}

There has always been consensus that barrel-related circuits process lemniscal inputs that transit through the thalamic barreloids, but the origin of vibrissal input to septa has long remained an unsettled issue. A recurring proposal, which principally relies on the fact that Po projects to the dysgranular regions of the barrel cortex (Lu and Lin, 1993), was that septarelated circuits process paralemniscal inputs that arise from the interpolaris division of the brainstem trigeminal complex. Although this proposal was inconsistent with the fact that Po neurons responded more tardily than septal cells to vibrissal stimuli (Armstrong-James and Fox, 1987; Diamond et al., 1992; Brumberg et al., 1999), it kept lingering in the literature for want of a better explanation. The recent discovery of a multiwhisker pathway that transits through the head of the 

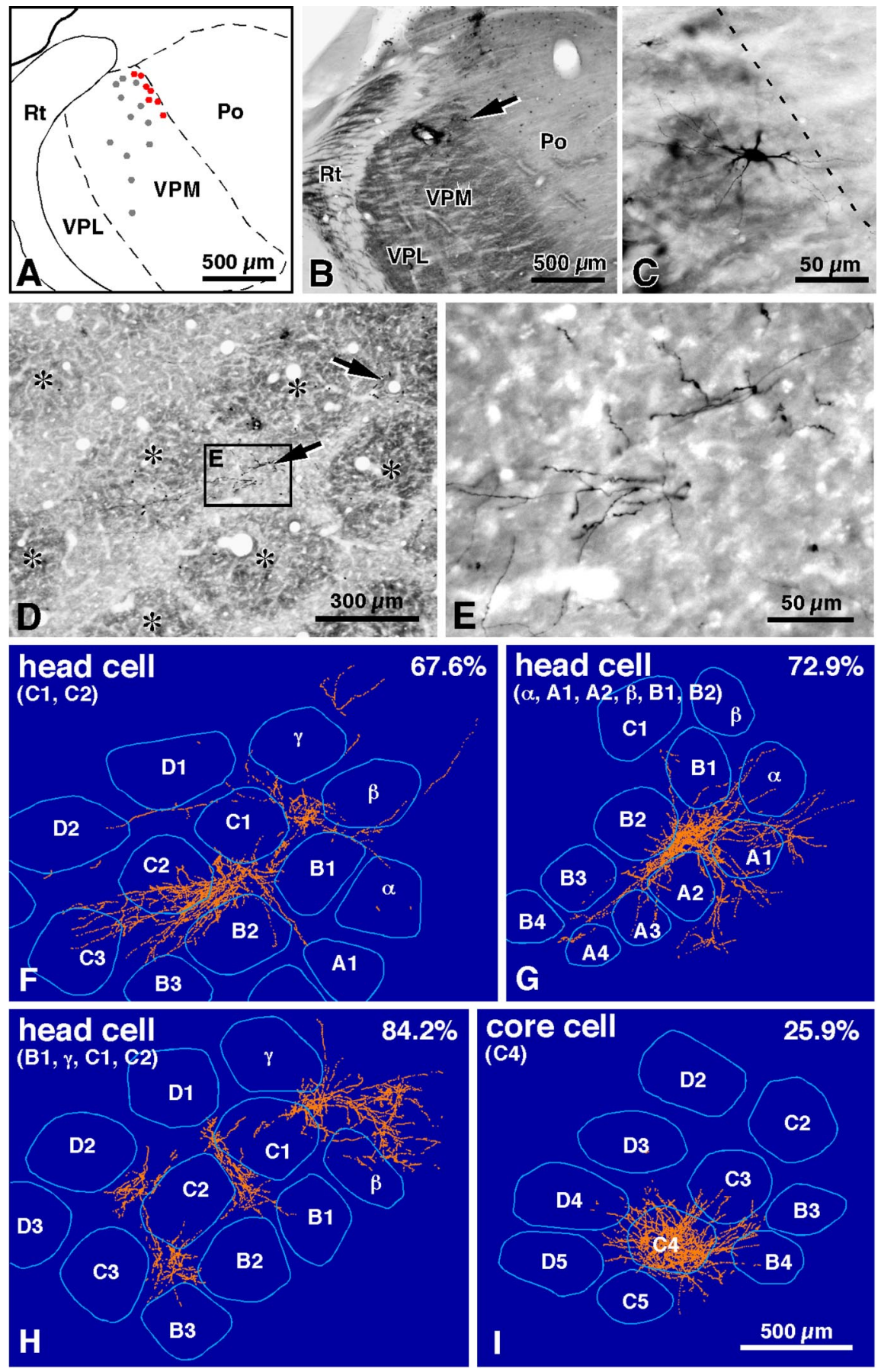

Figure 3. Cells in the head of the barreloids project to septa. $A$, Nineteen barreloid cells were juxtacellularly labeled with BDA 7 cells responded to multiple vibrissae (red dots) and 12 neurons responded to a single vibrissa (gray dots). $\boldsymbol{B}, \boldsymbol{C}$, Photomicrographs provide low- and high-power views of a multiwhisker unit labeled in the head of a barreloid. $\boldsymbol{D}, \boldsymbol{E}$, Tangential section through the barrel field ( $\boldsymbol{D}$; asterisks, barrels) shows some of the axonal branches of the unit labeled in $\boldsymbol{C}$; arrows point to main axonal branch points, and the boxed area is displayed at higher magnification in $\boldsymbol{E}$. $\boldsymbol{F}$-I provide ensemble views of the boutons given off in layer 4 by three head cells $(\boldsymbol{F}-\boldsymbol{H})$ and one core cell $(\boldsymbol{I})$. The terminal field in $\boldsymbol{F}$ belongs to the head cell shown in $\boldsymbol{C}$. Vibrissae that compose the receptive field of each cell are indicated in the upper left corner, whereas numbers in the upper right corner indicate the percentage of boutons in septa.

barreloids (Urbain and Deschênes, 2007) raised the possibility that septal cells derive their receptive field input from this subpopulation of VPM neurons. This is precisely what the present study demonstrates. Septal cells maintain their multiwhisker receptive field and response properties after brainstem lesion that prevents vibrissal input from activating the paralemniscal pathway. Conversely, lesion of the lemniscal pathway abolishes nearly completely vibrissal responses throughout barrel cortex. Furthermore, the labeling of single VPM cells revealed that multiwhisker cells in the head of barreloids project to the septal regions of the barrel cortex. These results thus provide the missing piece of evidence for a separate stream within the lemniscal pathway that conveys vibrissal information to septal columns of the barrel cortex.

Organization of the lemniscal pathway The lemniscal pathway in rodents is the primary source of vibrissal information to the VPM. This pathway arises from the $\mathrm{PrV}$, and comprises two populations of trigeminothalamic neurons: small-sized monowhisker cells that reside in whiskerrelated cellular aggregates called barrelettes, and large-sized multiwhisker neurons whose soma is located in the septa between the barrelettes (Henderson and Jacquin, 1995; Lo et al., 1999). The former population projects to the head and core of the barreloids, whereas the latter projects to both Po and the head of barreloids (Veinante and Deschênes, 1999). Thus, in contrast with cells forming the "core" of a barreloid whose receptive field is dominated by a single vibrissa, those situated in the "head" of a barreloid respond equally well to multiple vibrissae (see also Ito, 1988; Sugitani et al., 1990; Urbain and Deschênes, 2007). The distinction between the two populations of barreloid cells is particularly obvious in deeply anesthetized animals where head cells maintain multiwhisker receptive field whereas core cells become strictly monowhiskerresponsive. Head cells further differ from core cells in that they receive input from specific sets of reticular thalamic and corticothalamic fibers (Bourassa et al., 1995; Desîlets-Roy et al., 2002), and they are also contacted by corticothalamic input from lamina 6 of the vibrissa motor cortex (Urbain and Deschênes, 2007). The present demonstration that head cells project to septal columns is to be considered with the fact that septal columns provide the bulk of corticocortical input to vibrissa motor cortex (Alloway et al., 2004), which in turn projects back to the head of the barreloids. Thus the projection of head cells to the septa points to a potential link between head cells signaling and whisker motion (see Alloway, 2008 for the anatomical links between septal columns and motor circuitry). This raises the possibility that head cells might be the ones that convey information about whisker motion during free whisking (i.e., whisking in the absence of contact; Fee et al., 1997; Crochet and Petersen, 2006). 


\section{Receptive field synthesis in layer 4 cells}

It is now well established that two classes of lamina 4 cells exhibit multiwhisker receptive field in deeply anesthetized animals: regular-spiking septal cells and fast-spiking neurons (i.e., local circuit cells) that reside in both barrels and septa (Simons, 1995). It is also agreed that, given their short latency of activation by sensory stimuli, both populations of cells derive their multiwhisker input from the thalamus (Brumberg et al., 1999; Bruno and Simons, 2002; Swadlow and Gusev, 2002; Bruno and Sakmann, 2007). The present results indicate that head cells are the primary source of multiwhisker input to septa. However, given that the terminal field of most core cells also invades surrounding septa, one cannot exclude the possibility that some septal neurons derive their multiwhisker receptive field from inputs of core cells that reside in adjacent barreloids. Yet, this would hold only for septal neurons that respond to two to four whiskers that are contiguous on the mystacial pad, but not for the vast majority (i.e., 70\%, 27 of 38 cells in the present study) whose receptive field included noncontiguous vibrissae pertaining to the same arc or row of whisker representation (see Fig. S2, available at www. jneurosci.org as supplemental material).

Our data show that head cells distribute $\sim 25 \%$ of their terminals in barrels; yet, in our recording conditions regular spiking barrel cells were strictly monowhisker responsive. This suggests that the collective input of head cells to a barrel might not represent a drive strong enough to excite these neurons in deeply anesthetized animals. Another, not exclusive possibility could be that head cells' projection into a barrel principally target fast-spiking neurons that have multiwhisker receptive field. Interestingly, a recent study (Simons et al., 2007) has raised the possibility that fast-spiking/inhibitory barrel cells, in contrast to excitatory barrel neurons, may be contacted by a subpopulation of broadly tuned, faster conducting barreloid cells that respond to whisker deflections more robustly and at shorter latency. Details about conduction velocity and response properties of head cells are not yet available, but cross-correlation analysis between the discharges of head cells and fast-spiking barrel cells could throw light on this issue. Yet, the projection patterns of barreloid cells may not all conform to those of the head and core cells we labeled. The possibility that some barreloid cells innervate multiple barrels has been suggested by the labeling study of Arnold et al. (2001), although evidence presented in that study did not rely on unequivocal identification of barrels and septa.

\section{The paralemniscal projection to barrel cortex}

After it was reported that septal columns receive input from Po (Lu and Lin, 1993), the actual impact of Po input on layer 4 septal neurons has never been clearly established. In fact, Po terminals distribute principally in layers $5 \mathrm{a}$ and 1 across both barrel and septal columns, suggesting that pyramidal cells in layer $5 \mathrm{a}$ throughout barrel cortex are the primary targets of the paralemniscal pathway (Ahissar et al., 2001). Thus, the usual association of barrel columns to the lemniscal and septal columns to the paralemniscal pathway appears questionable, as much as lesion of the paralemniscal pathway does not affect the magnitude and latency of vibrissal responses in layer 4 septal cells. This last result was expected because Po neurons respond more tardily than septal cells to whisker deflection (Armstrong-James and Fox, 1987; Diamond et al., 1992; Brumberg et al., 1999). It should be emphasized that the absence of short-latency responses in Po $(<10 \mathrm{~ms})$ cannot be attributed to anesthesia, because Po cells do not respond at short latency to passive whisker deflection in head-fixed alert animals (Masri et al., 2008). These results thus indicate that the role of Po and the associated cortical circuitry needs to be reappraised in a conceptual framework that takes into account behavioral contingencies related to motor activities, motivation, and expectations of the animal.

\section{References}

Ahissar E, Sosnik R, Bagdasarian K, Haidarliu S (2001) Temporal frequency of whisker movement. II. Laminar organization of cortical representations. J Neurophysiol 86:354-367.

Alloway KD (2008) Information processing streams in rodent barrel cortex: the differential functions of barrel and septal circuits. Cereb Cortex 18:979-989.

Alloway KD, Zhang M, Chakrabarti S (2004) Septal columns in rodent barrel cortex: functional circuits for modulating whisking behavior. J Comp Neurol 480:299-309.

Armstrong-James M, Fox K (1987) Spatiotemporal convergence and divergence in the rat SI barrel cortex. J Comp Neurol 263:265-281.

Arnold PB, Li CX, Waters RS (2001) Thalamocortical arbors extend beyond single cortical barrels: an in vivo intracellular tracing study in rat. Exp Brain Res 136:152-168.

Bokor H, Acsády L, Deschênes M (2008) Vibrissal responses of thalamic cells that project to the septal columns of the barrel cortex and to the second somatosensory area. J Neurosci 28:5169-5177.

Bourassa J, Pinault D, Deschênes M (1995) Corticothalamic projections from the cortical barrel field to the somatosensory thalamus in rats: a single-fibre study using biocytin as an anterograde tracer. Eur J Neurosci 7:19-30.

Brumberg JC, Pinto DJ, Simons DJ (1999) Cortical columnar processing in the rat whisker-to-barrel system. J Neurophysiol 82:1808-1817.

Bruno RM, Sakmann B (2006) Cortex is driven by weak but synchronously active thalamocortical synapses. Science 312:1622-1627.

Bruno RM, Simons DJ (2002) Feedforward mechanisms of excitatory and inhibitory cortical receptive fields. J Neurosci 22:10966-10975.

Crochet S, Petersen CC (2006) Correlating whisker behavior with membrane potential in barrel cortex of awaked mice. Nat Neurosci 9:608-610.

Desîlets-Roy B, Varga C, Lavallée P, Deschênes M (2002) Substrate for cross-talk inhibition between thalamic barreloids. J Neurosci 22:RC218:1-4.

Diamond ME, Armstrong-James M, Budway MJ, Ebner FF (1992) Somatic sensory responses in the rostral sector of the posterior group (POm) an in the ventral posterior medial nucleus (VPM) of the rat thalamus: dependence on the barrel field cortex. J Comp Neurol 319:66-84.

Fee MS, Mitra PP, Kleinfeld D (1997) Central versus peripheral determinants of patterned spike activity in rat vibrissa cortex during whisking. J Neurophysiol 78:1144-1149.

Friedberg MH, Lee SM, Ebner FF (1999) Modulation of receptive field properties of thalamic somatosensory neurons by the depth of anesthesia. J Neurophysiol 81:2243-2252.

Henderson TA, Jacquin MF (1995) What makes subcortical barrels? In: Cerebral cortex, vol 12 (Jones EG, Diamond IT, eds), pp 123-187. New York: Plenum.

Ito M (1988) Response properties and topography of vibrissa-sensitive VPM neurons in the rat. J Neurophysiol 60:1181-1197.

Kim U, Ebner FF (1999) Barrels and septa: separate circuits in rat barrels field cortex. J Comp Neurol 408:489-505.

Lavallée P, Urbain N, Dufresne C, Bokor H, Acsády L, Deschênes M (2005) Feedforward inhibitory control of sensory information in higher-order thalamic nuclei. J Neurosci 25:7489-7498.

Lo FS, Guido W, Erzurumlu RS (1999) Electrophysiological properties and synaptic responses of cells in the trigeminal principal sensory nucleus of postnatal rats. J Neurophysiol 82:2765-2775.

Lu SM, Lin RC (1993) Thalamic afferents of the rat barrel cortex: a lightand electron-microscopic study using Phaseolus vulgaris leucoagglutinin as an anterograde tracer. Somatosens Mot Res 10:1-16.

Masri R, Bezdudnaya T, Trageser JC, Keller A (2008) Encoding of stimulus frequency and sensor motion in the posterior medial thalamic nucleus. J Neurophysiol 100:681-689.

Paxinos G, Watson W (1998) The rat brain in stereotaxic coordinates, Ed 4. London: Academic.

Pierret T, Lavallée P, Deschênes M (2000) Parallel streams for the relay of vibrissal information through thalamic barreloids. J Neurosci 20:7455-7462. 
Pinault D (1996) A novel single-cell staining procedure performed in vivo under electrophysiological control: morpho-functional features of juxtacellularly labeled thalamic cells and other central neurons with biocytin or neurobiotin. J Neurosci Methods 65:113-136.

Simons DJ (1995) Neuronal integration in the somatosensory whisker/barrel cortex. In: Cerebral cortex, vol 12 (Jones EG, Diamond IT, eds), pp 263-297. New York: Plenum.

Simons DJ, Carvell GE, Kyriazi HT, Bruno RM (2007) Thalamocortical conduction times and stimulus-evoked responses in the rat whisker-tobarrel system. J Neurophysiol 98:2842-2847.

Sugitani M, Yano J, Sugai T, Ooyama H (1990) Somatotopic organization and columnar structure of vibrissae representation in the rat ventrobasal complex. Exp Brain Res 81:346-352.
Swadlow HA, Gusev AG (2002) Receptive-field construction in cortical inhibitory interneurons. Nat Neurosci 5:403-404.

Trageser JC, Keller A (2004) Reducing the uncertainty: gating of peripheral inputs by zona incerta. J Neurosci 24:8911-8915.

Urbain N, Deschênes M (2007) A new pathway of vibrissal information processing modulated by the motor cortex. J Neurosci 27:12407-12412.

Veinante P, Deschênes M (1999) Single- and multi-whisker channels in the ascending projections from the principal trigeminal nucleus in the rat. J Neurosci 19:5085-5095.

White EL, Weinfeld E, Lev DL (2004) Quantitative analysis of synaptic distribution along thalamocortical axons in adult mouse barrels. J Comp Neurol 479:56-69. 